\title{
Investigation of the obscuring circumnuclear torus in the active galaxy Mrk231
}

\author{
Hans-Rainer Klöckner ${ }^{1,2}$, Willem A. Baan ${ }^{2}$ and Michael A. Garrett ${ }^{3}$ \\ 1. Kapteyn Astronomical Institute, University of Groningen, 9700 AV, Groningen, The \\ Netherlands \\ 2. ASTRON, P.O.Box 2, 7990 AA, Dwingeloo, The Netherlands \\ 3. JIVE, Joint Institute for VLBI in Europe, PO Box 2, 7990 AA Dwingeloo, The Nether- \\ lands
}

published in Nature Vol. 421, 821-823, 20 February 2003

Active galaxies are characterized by prominent emission from their nuclei. In the unified view of active galaxies, the accretion of material onto a massive compact object - now generally believed to be a black hole - provides the fundamental power source (Rees, 1984). Obscuring material along the line of sight can account for the observed differences in nuclear emission (Krolik \& Begelman, 1988; Krolik, 1999), which determine the classification of AGN (for example, as Seyfert 1 or Seyfert 2 galaxies). Although the physical processes of accretion have been confirmed observationally (Greenhill et al., 1995; Gallimore et al., 1997), the structure and extent of the obscuring material have not been determined. Here we report observations of powerful hydroxyl $(\mathrm{OH})$ line emissions that trace this obscuring material within the circumnuclear environment of the galaxy Markarian 231. The hydroxyl (mega)-maser emission shows the characteristics of a rotating, dusty, molecular torus (or thick disk) located between 30 and 100 pc from the central engine. We now have a clear view of the physical conditions, the kinematics and the spatial structure of this material on intermediate size scales, confirming the main tenets of unification models.

Observations of powerful molecular water-vapour and hydroxyl megamaser emissions, which are a million times more luminous than their galactic counterparts (Baan, 1985), have provided unique information about distinct regions within galactic nuclei. Water-vapour masers can map out the central few parsecs of an AGN, tracing the rotation of nuclear accretion disks and the state of molecular material surrounding the radio jets (Malonev, 2002). The inferred geometry and thickness of these disks, however, cannot account for the observed obscuration signature and the radiation patterns seen in active 
galactic nuclei (AGN) (Malonev, 2002; Herrnstein et al., 1998). On the other hand, the hydroxyl masers in galactic nuclei are spread across hundreds of parsecs (Diamond et al., 1999; Lonsdale et al., 1998; Pihlström et al., 2001). Their tight correlation with the infrared emission (Baan, 1989) indicates a strong association with the dusty obscuring material along the line of sight towards the nucleus (Krolik \& Begelman, 1988; Krolik, 1999). To study the distribution of the hydroxyl megamaser emission with respect to the nuclear engine at a resolution of tens of parsecs in Mrk 231, we performed very-longbaseline interferometry (VLBI) radio observations with the European VLBI Network (EVN). Mrk 231 is the most luminous infrared galaxy in the local universe (distance of $172 \mathrm{Mpc}$ ), and is undergoing a merger as indicated by the high dust content and the prominent tidal tails observed at optical wavelengths. The nuclear power source has been classified (Baan et al., 1998) as a Seyfert 1, which in the unification scheme would suggest that the observer has a direct, and almost unobscured, line-of-sight view into the broad-line emission region at parsec scales. The location of the nuclear engine on similar scale sizes is traced by the radio outflow (Ulvestad et al., 1999b), which extends to almost a hundred parsecs (Ulvestad et al., 1999a).

The EVN data reveal an $\mathrm{OH}$ emission structure with an east-west extent of $130 \mathrm{pc}$ that is straddling a north-south elongated radio continuum emission (Fig. (1). This continuum emission has been further resolved into a lobe-corelobe structure (Ulvestad et al., 1999a) of $80 \mathrm{pc}$ at position angle $\mathrm{PA}=8^{\circ}$, which is found to be misaligned by $57^{\circ}$ with the core radio structure on parsec scales (Ulvestad et al., 1999b). The emission spectrum integrated across the whole $\mathrm{OH}$ emitting region displays the two main hydroxyl lines at 1667 and $1665 \mathrm{MHz}$ showing a line-width of around $200 \mathrm{~km} \mathrm{~s}^{-1}$ (full-width at halfmaximum, FWHM) at a centroid velocity of $12610 \mathrm{~km} \mathrm{~s}^{-1}$ (Fig. 21). The EVN data and also the intermediate-scale MERLIN (Richards et al., 2000) observations account for only half of the integrated line emission seen by the Westerbork Synthesis Radio Telescope (WSRT; see Figure 2) and previous singledish measurements (Baan, 1985). This missing emission component must therefore originate within the resolution gap between the EVN and WSRT observations, in an extended region between $190 \mathrm{pc}$ and $11 \mathrm{kpc}$ of the nucleus. This component is probably associated with the nuclear continuum emission (Tavlor et al., 1999) and the CO(1-0) line emission (Brvant \& Scoville, 1996; Downes \& Solomon, 1998). Figure 3 (top panel) presents the spatial velocity distribution, which is similar for each of the two hydroxyl lines and which has 
an overall velocity shift of about $160 \mathrm{~km} \mathrm{~s}^{-1}$ from the northwest towards the southeast. The velocity width of the emission lines has an average value of about $70 \mathrm{~km} \mathrm{~s}^{-1}$ (FWHM) across the northern part of the radio continuum emission. On the other hand, there is also a distinct $\mathrm{OH}$ emission region at the northwestern edge of the continuum emission that has a velocity similar to that seen towards the centre of the radio core, and which has a distinctly higher velocity width of $85 \mathrm{~km} \mathrm{~s}^{-1}$ (Figure 3 ; middle panel).

The nature of the $\mathrm{OH}$ emission observed in the nucleus of Mrk 231 adds a new component to the hierarchical structure of this system, and complements the information obtained with other tracers at lower resolution. We find that the $\mathrm{OH}$ velocity field with its symmetry axis at $\mathrm{PA}=34^{\circ}$ does not agree with that of the kiloparsec-scale $\mathrm{CO}(1-0)$ structure (Bryant \& Scoville, 1996; Downes \& Solomon, 1998) having a PA $=0^{\circ}$. Neither does it agree with the large-scale radio outflow $\left(\mathrm{PA}=8^{\circ}\right)$, or the more compact $\mathrm{CO}(2$ 1) distribution (Downes \& Solomon, 1998) extending up to $850 \mathrm{pc}$ at $\mathrm{PA}=$ $18^{\circ}$. However, the $\mathrm{OH}$ velocity field is in agreement with that of the lineof-sight HI absorption (Carilli et al., 1998) at PA $=27^{\circ}$. The HI absorption is detected against a diffuse radio-emitting halo with an extent of about $350 \mathrm{pc}$ and an orientation of $\mathrm{PA}=22^{\circ}$. This diffuse radio halo, which is possibly associated with a circumnuclear starburst (Tavlor et al., 1999), could then also trace the dense and opaque interstellar medium of a disk or torus (Carilli et al., 1998) inclined at $56^{\circ}$. The integrated HI velocity dispersion (Carilli et al., 1998) of $193 \pm 25 \mathrm{~km} \mathrm{~s}^{-1}$ agrees well with that of the hydroxyl lines (Fig. 2). This suggests that the $\mathrm{OH}$ emission indeed originates in the central regions of a circumnuclear rotating disk or torus having an outer structure that is traced by the $\mathrm{CO}(2-1)$ emission.

The energy source responsible for the excitation of the $\mathrm{OH}$ molecules is the extreme far-infrared radiation (Baan, 1989) field in Mrk 231. The observed far-infrared spectral temperature of around $43 \mathrm{~K}$ provides the ideal pumping conditions for the $\mathrm{OH}$ clouds, which could range in size from $10 \mathrm{pc}$ up to 100 pc (Henkel et al., 1987; Randell et al., 1995). The ratio of the intensity of the two maser lines varies rather smoothly across the entire emission structure and has an average value of 1.8 , as expected for local temperature equilibrium (LTE). This would indicate that the observed emission lines are optically thin and are mostly unsaturated masers. Assuming that the diffuse radio continuum, which serves as a background for the nuclear HI absorption, also serves as a background for the observed $\mathrm{OH}$ masers, this emission could 
be explained in terms of exponential amplification with a maximal gain of about 2.2 within classical OH maser-models (Baan et al., 1982; Baan, 1985). However, extreme values for the line ratio have been found in the discrepant northwestern region discussed earlier in this text. This region must have a completely different pumping environment than the rest of the structure. The unusual blue-shifted velocity, the slightly higher velocity dispersion, and the line ratios of this discrepant region are all consistent with the theoretically predictions (Baan, 1985; Randell et al., 1995; Wada \& Norman, 2002; Baan, 1985) for an interaction region between the radio outflow and the molecular environment.

The molecular environment described above for the nuclear region of Mrk 231 is very similar to the dust structures found in active galaxies such as NGC 4261 using the Hubble Space Telescope (van Langevelde et al., 2000). We have applied an axisymmetric torus model with a circular cross-section to the EVN data, in order to determine the structural boundaries in the nuclear region. The compact molecular structure surrounding the nucleus is misaligned with the large-scale molecular disk of the galaxy by $34^{\circ}$. The radius of the inner cavity of the torus would be about $30 \mathrm{pc}$, as suggested by the location of the northwestern interaction region. The outer edge of the torus spans a diameter of $200 \mathrm{pc}$, which is based on the $4 \mathrm{mJy}$ contours of the diffuse radio emission (Carilli et al., 1998) and on far-infrared blackbody estimates (Downes \& Solomon, 1998). This region also serves as a radio background for the $\mathrm{OH}$ amplification process. These parameters result in a torus structure with a radius of $65 \mathrm{pc}$ and a thickness of $70 \mathrm{pc}$, which accounts for an obscuration angle of $60^{\circ}$ centered on the plane of the torus and which is representative of values found for other galaxies (Schmitt et al., 2001). The orientation and the shape of the extended radio contours and the symmetry axis of the velocity field indicate that the torus is tilted upward from the line of sight by $56^{\circ}$, and is rotated anticlockwise by $35^{\circ}$. This inferred model for the torus has been displayed as a wire-structure representation and is presented in Figure 3 (bottom panel). The nuclear radiation cones with opening angles of $60^{\circ}$, assumed to be similar to those seen in M 87 (Junor et al., 1999), represent the directions with an unobscured view of the nucleus. The radio outflow follows a twisted path within these cones, starting at the nucleus (at PA $=65^{\circ}$ ) and ending up as a double source aligned with the symmetry axis of the outer disk (at PA $=8^{\circ}$ ) (Ulvestad et al., 1999b. a). As a result, this parameterization indicates that the nuclear accretion disk, 
that collimates the jet outflow inside the central cavity, is actually misaligned by $30^{\circ}$ with the plane of the molecular torus.

In reality, the proposed torus structure does not need to have a circular cross-section. In addition, it will most probably be wrapped inside a cocoonlike surface region with a higher temperature, and will have the same outer extent of $460 \mathrm{pc}$ as the diffuse radio emission and the HI absorbing gas (Carilli et al., 1998). However, the representation of the inner part of this rotating dusty and molecular torus in Mrk 231 is consistent with (all) current models of galactic nuclei and theoretical investigations (Krolik, 1999), and supports the unification schemes for AGN. Whether a different orientation of the torus and the nuclear accretion disk represents a special case or is a general characteristic of active nuclei needs to be investigated with VLBI observations of other megamaser galaxies hosting a similar nuclear power source.

\section{Acknowledgments}

We thank C. Carilli for providing a map of the diffuse continuum structure in Mrk 231. H.-R.K thanks O. Möller for advice on programming in OpenGL software. The European VLBI Network is a joint facility of European, South African, and Chinese radio astronomy institutes funded by their national research councils. The Westerbork Synthesis Radio Telescope is operated by the ASTRON (Netherlands Foundation for Research in Astronomy) with

support from the Netherlands Foundation for Scientific Research NWO.

Correspondence and requests for material should be addressed to H.-R. K.(email: hrkloeck@astro.rug.nl).

\section{References}

Baan W.A.: 1985, Nat 315, 26

Baan W.A.: 1989, ApJ 338, 804

Baan W.A., Salzer J.J., Lewinter R.D.: 1998, ApJ 509, 633

Baan W.A., Wood P.A.D., Haschick A.D.: 1982, ApJ Lett. 260, L49

Bryant P.M., Scoville N.Z.: 1996, ApJ 457, 678 
Carilli C.L., Wrobel J.M., Ulvestad J.S.: 1998, AJ 115, 928

Diamond P.J., Lonsdale C.J., Lonsdale C.J., Smith H.E.: 1999, ApJ 511, 178

Downes D., Solomon P.M.: 1998, ApJ 507, 615

Gallimore J.F., Baum S.A., O'Dea C.P.: 1997, Nat 388, 852

Greenhill L.J., Jiang D.R., Moran J.M., et al.: 1995, ApJ 440, 619+

Henkel C., Guesten R., Baan W.A.: 1987, A\&GA 185, 14

Herrnstein J.R., Moran J.M., Greenhill L.J., Blackman E.G., Diamond P.J.: 1998, ApJ 508, 243

Junor W., Biretta J.A., Livio M.: 1999, Nat 401, 891

Krolik J.H.: 1999, Active galactic nuclei : from the central black hole to the galactic environment. Active galactic nuclei : from the central black hole to the galactic environment /Julian H. Krolik. Princeton, N. J. : Princeton University Press, c1999.

Krolik J.H., Begelman M.C.: 1988, ApJ 329, 702

Lonsdale C.J., Lonsdale C.J., Diamond P.J., Smith H.E.: 1998, ApJ Lett. 493, L13

Maloney P.R.: 2002, Publications of the Astronomical Society of Australia 19, 401

Pihlström Y.M., Conway J.E., Booth R.S., Diamond P.J., Polatidis A.G.: 2001, A\&A 377, 413

Randell J., Field D., Jones K.N., Yates J.A., Gray M.D.: 1995, A\&3A 300, 659

Rees M.J.: 1984, ARA\&A 22, 471

Richards A., Cohen, Cole, et al.: 2000, In: Galaxies and their Constituents at Highest Angular Resolution IAU Symposium, vol. 205, Astronimical Society of the Pacific, San Francisco, c2001. 
Schmitt H.R., Antonucci R.R.J., Ulvestad J.S., et al.: 2001, ApJ 555, 663

Taylor G.B., Silver C.S., Ulvestad J.S., Carilli C.L.: 1999, ApJ 519, 185

Ulvestad J.S., Wrobel J.M., Carilli C.L.: 1999a, ApJ 516, 127

Ulvestad J.S., Wrobel J.M., Roy A.L., et al.: 1999b, ApJ Lett. 517, L81

van Langevelde H.J., Pihlström Y.M., Conway J.E., Jaffe W., Schilizzi R.T.: 2000, A\&BA 354, L45

Wada K., Norman C.A.: 2002, ApJ Lett. 566, L21 


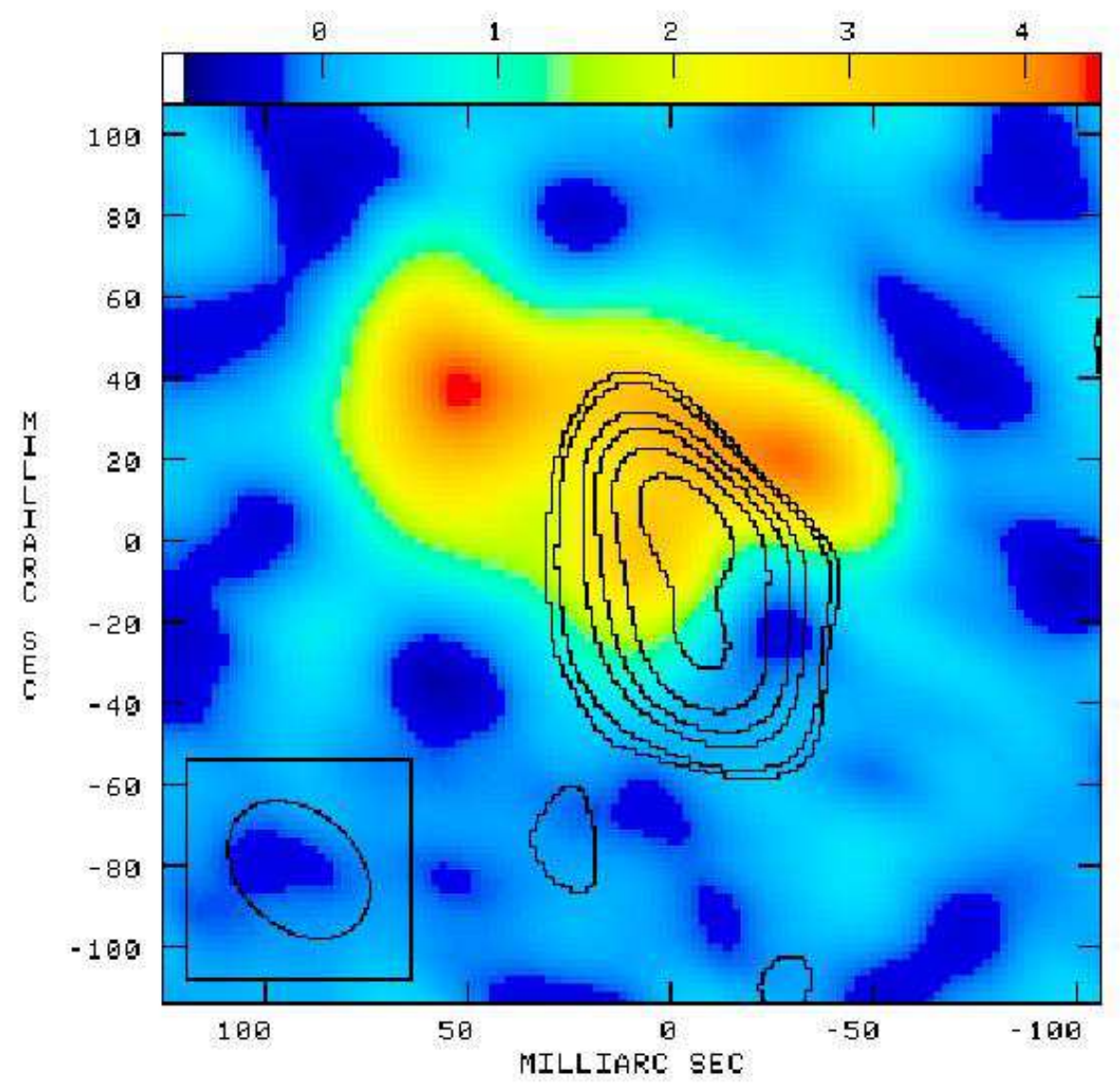

Figure 1: Hydroxyl and radio-continuum emission in the nucleus of Mrk 231.

The integrated $\mathrm{OH}$-line emission in pseudo-color (in mJy per beam) is superimposed on the nuclear continuum emission in contours (peak $39 \mathrm{mJy}$ per beam). The contour levels are a geometric progression in factors of the square root of 2 starting at 4 mJy per beam. These EVN observations of Mrk 231 were made on September 1999 for $12 \mathrm{hrs}$ in dual-polarization phasereferencing mode at $1599 \mathrm{MHz}$ and with 256 spectral channels across a total bandwidth of $8 \mathrm{MHz}$. The synthesized beam is 39 mas $\times 28$ mas, where 1 mas corresponds to a size of $0.83 \mathrm{pc}$ at the distance of Mrk 231 (172 Mpc by assuming $\mathrm{q}_{0}=0.5 \mathrm{~km} \mathrm{~s}^{-1}$ and $\mathrm{H}_{0}=75 \mathrm{~km} \mathrm{~s}^{-1} \mathrm{Mpc}^{-1}$ ). The data has been correlated at the EVN correlator at the Joint Institute for VLBI in Europe (JIVE), and later transferred to the AIPS packages for calibration of the complex visibilities. The integrated line emission image has been obtained by averaging over the velocity range of the $\mathrm{OH}$ lines, after subtraction of the radio continuum, obtained by averaging the channels without line emission. 


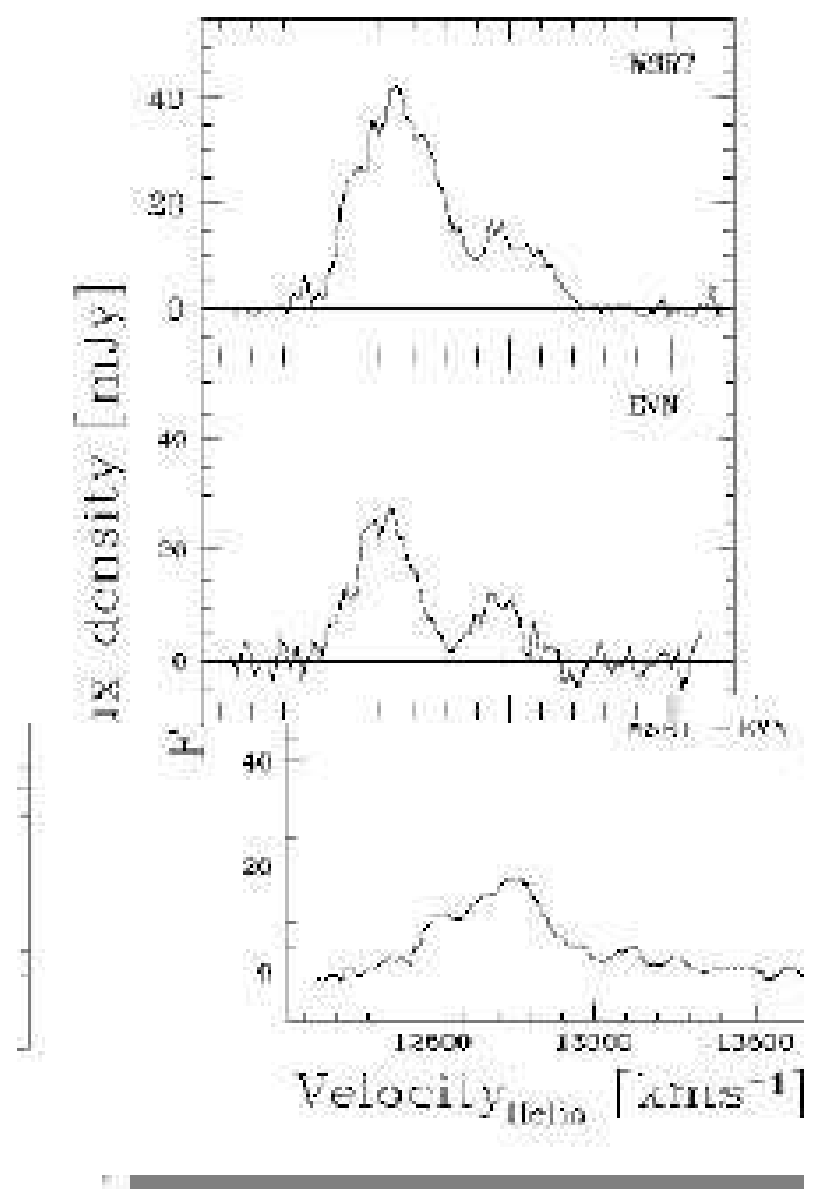

Figure 2: Hydroxyl emission spectra of Mrk 231 traced at different scale sizes.

The top panel shows the WSRT spectrum taken at 14 arcsec resolution, the middle panel displays the spectrum for the EVN observations at 39 mas resolution, and the bottom panel displays the difference spectrum of both observations. The emission line features show both $\mathrm{OH}$ main lines at 1667 and $1665 \mathrm{MHz}$ that are offset by $365 \mathrm{~km} \mathrm{~s}^{-1}$; the velocity scale refers to the $1667 \mathrm{MHz}$ emission line by assuming a heliocentric velocity of $\mathrm{V}_{\text {center }}$ $=12650 \mathrm{~km} \mathrm{~s}^{-1}$ based on the $\mathrm{CO}(1-0)$ centre velocity (Downes \& Solomon, 1998). The spectral resolution is $18 \mathrm{~km} \mathrm{~s}^{-1}$ per channel. The $\mathrm{OH}$ line width in the EVN data is $214 \pm 11 \mathrm{~km} \mathrm{~s}^{-1}$ (FWHM), which is $70 \mathrm{~km} \mathrm{~s}^{-1}$ less than for the WSRT data. The residual emission line in the bottom frame counts for $44 \%$ of the $\mathrm{OH}$ emission in Mrk 231 and is systematically redshifted by around $45 \mathrm{~km} \mathrm{~s}^{-1}$. Similar flux discrepancies, but with no distinct velocity offset, have been found for other $\mathrm{OH}-\mathrm{MM}$ galaxies studied at high resolution (Diamond et al,, 1999; Lonsdale et al,, 1998; Pihlström et al, 2001). Similar velocity offsets have also been found for the $\mathrm{CO}(2-1)$ emission (Brvant \& Scoville, 1996) and the HI absorption in Mrk 231 (Carilli et al., 1998). 

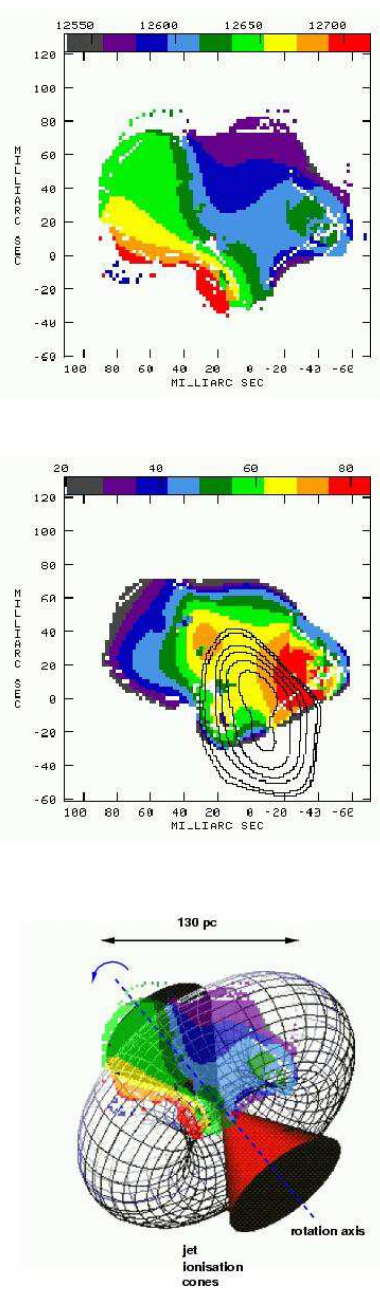

Figure 3: The circum-nuclear kinematics in Mrk 231.

Top panel - The velocity field of the $\mathrm{OH} 1667 \mathrm{MHz}$ emission in the EVN data is shown in pseudo-colors. The spatial resolution is the same as for Fig. 1 and the velocity scale is the same as in Fig. 2. The overall velocity pattern reveals an east-west velocity gradient of $1.41 \mathrm{~km} \mathrm{~s}^{-1}$ per parsec over the entire $\mathrm{OH}$ emission such that the northwestern edge of the torus moves towards the observer.

Middle panel - The line width of the $1667 \mathrm{MHz}$ emission lines (in $\mathrm{km} \mathrm{s}^{-1}$ ) is superimposed on the nuclear radio continuum emission in contours displayed in a similar way as in Fig. 1. The diagram shows that the velocity dispersion varies significantly across the emission region. The velocity dispersion structure shows several distinct regions but with the highest values to be found at the western edge of the radio continuum source.

Bottom panel - The inferred model of the nuclear torus in Mrk 231 is displayed as a wire diagram with symmetric ionization cones. This model takes into account all large-scale characteristics of the nuclear radio emission and the $\mathrm{OH}$ emission. The symmetry axis of the torus has been inclined upwards by $56^{\circ}$, and then rotated anticlockwise by $35^{\circ}$. The molecular material moves from top-right to bottom-left (northwest to southeast). The virial estimates of the central mass concentration is $7.2 \pm 3.8 \times 10^{7} \mathrm{M}$ 